\title{
A Steepest Descent Relocation Algorithm for Placement of Sinks in A Sensor Network
}

\author{
Mehdi Kalantari, Mark Shayman \\ Department of Electrical and Computer Engineering, University of Maryland \\ \{mehkalan, shayman\}@eng.umd.edu
}

\begin{abstract}
In this paper we use an information flow model for placement of traffic sinks in a wireless sensor network. Our mathematical model translates a communication network composed of countably many sensors into a continuum model described by a continuous vector field. This vector field models flow of information in a sensor network, and its magnitude is the intensity of the communication activity, and its orientation is the direction in which the traffic is forwarded. We use this formulation for design of a sensor network in which the traffic generated by the wireless sensors needs to be routed to one of the multiple traffic sinks in different locations of the network. We show that the optimal vector field satisfies a set of PDEs similar to Maxwell's equations in electrostatics, with Neumann boundary conditions. We prove that the orientation of the vector field at the location of each sink is the direction of the steepest descent of the total communication cost function of the network. This direction corresponds to the direction of force in an analogous electrostatics problem. We use this result in order to introduce iterations that relocate sinks and show that in the optimal placement of sinks, the value of the vector field is zero at the location of each sinks.
\end{abstract}

\section{INTRODUCTION}

Wireless sensor networks have been studied extensively in recent years. Such networks are made up of several hundred to several thousand sensors distributed in a geographical area. There are many applications for such networks including military, environment monitoring, surveillance, agriculture and home applications. Generally, sensors use radio frequency channels for communicating, and it is desired to collect the data acquired by all sensors at a few specific destinations in the network for processing. Such stations are known as sinks or fusion centers. For the purpose of communication to the traffic sinks, the sensors relay the packets of each other in a multi-hop way.

The idea of using a vector field to model information flow in a wireless network, which is inspired by electrostatic, was introduced in our former works [1,2]. This work was followed by $[3,4]$, where the author showed that the methodology leads to an optimal deployment of sensor nodes. In this methodology a traffic density vector field models transportation of traffic in a wireless network. The vector field has two components at every location of the network: a magnitude that represents the density of communication at that location and an orientation that gives the direction to which the traffic is forwarded. The model is best suitable in situations where the sensors in the network are distributed with a high enough density so that the routes from individual sensors to the traffic sinks can

This work was partially supported by AFOSR under grant F496200210217 and and NSF under grant CNS-0519554. be well approximated by the flux lines of the vector field passing through sensors. The first property of the vector field is that it satisfies a partial differential equation (PDE) that guarantees the traffic of all sources is transported to the sinks. This constraint is incorporated into our model through the divergence of the vector field and represents flow conservation law in conventional networks. Additionally, the vector field satisfies a set of Neumann boundary conditions, which state that the vector field has a zero component in the direction normal to the boundary of the network.

We show that the divergence property of the traffic vector field and the boundary conditions do not specify the vector field uniquely. Therefore, we have a degree of freedom to optimize a measure of performance in the network. For this purpose, we minimize an integral of a quadratic form of the vector field over the network area. This cost function has many interesting properties. First, it tries to minimize the total communication burden required to send the traffic created by the sources to the destinations. Second, it spreads the communication load of the network over the space, so that we make the best use of the available space in the network. This reduces the mutual interference between the wireless nodes, and increases the overall network throughput. Additionally, in [3] it is shown that minimizing the quadratic cost function minimizes the total number of wireless nodes required to transport the traffic generated by the sources to the sinks. The optimization of the quadratic cost function leads to a second PDE, which states that the curl of the vector field should be zero. While we showed this property mathematically, it has an interesting intuitive interpretation: since the curl of a vector field is the density of its rotation, the "zero curl" property states that the optimal traffic forwarding scheme based on the vector field does not introduce any routing loops.

The solution of our optimization problem is found as the joint solution of a set of PDEs that involve the divergence property, the zero curl property, and the Neumann type boundary conditions. The existence and uniqueness of the solution for this system of PDEs is guaranteed based on the mature literature on the PDEs [5,6]. An observation that helps us solve the PDEs numerically is the fact that a zero curl vector field (also known as an irrotational or conservative vector field) can be written as the gradient of a potential field. Using this property, we can translate the set of two first-order PDEs into a single second-order PDE that involves the potential function. The optimal solution of the problem, this potential function satisfies the classical Poisson's equation.

An interesting result of our methodology is its analogy to 
the theory of electrostatics in classical physics [7-9]. We will observe that the traffic vector field is analogous to the electric field intensity, the divergence and zero curl properties are analogous to the Maxwell's equations, the potential function is analogous to the electric potential, the traffic sources are analogous to a distributed density of positive charges, the traffic sinks are analogous to negative point charges, and the quadratic cost function is analogous to the energy in an electrostatic equilibrium. Our proposed tool based on vector calculus provides many different mathematical tools to address the design issues of sensor networks:

- Optimal load assignment: The vector field gives the optimal amount of traffic that needs to be routed to each one of the traffic sinks. In an optimal assignment of the network traffic load to the traffic sinks, the value of the potential function at the location of all the traffic sinks should be equal.

- Optimal partitioning of the network: the methodology gives an optimal partitioning of the network into several territories, each assigned to one of the traffic sinks.

- Placement of the traffic sinks: the vector field gives direction over which each traffic sink should be relocated in order to achieve the steepest descent of the cost function. We show that this direction is analogous to the direction of the applied force on a charged particle in electrostatics. This issue is the focus of this paper.

The remainder of this paper is organized as follows: in Section II we give the background and summary of our prior results. In Section III. We use the method to find the direction of relocation of sinks that results in the steepest descent in the cost function. We show a step by step summary of the design algorithm in Section IV. Some illustrative examples and numerical experiments are given in Section $\mathrm{V}$, and the paper concludes in Section VI.

\section{INFORMATION FlOW AS A Vector Field}

In this section, we give an overview of our previous work on routing in wireless networks by using vector fields. We briefly state our results here; more complete presentations of these results can be found in $[1,2,10]$.

Let us assume that we have a network in an area $A$ on the plane, with $M$ information sinks that receive the traffic of all the wireless nodes. The $i^{t h}$ sink is located at position $\mathbf{z}_{i}=$ $\left(x_{i}, y_{i}\right)$. The density of traffic sources is given by the spatial function denoted by $r(\mathbf{z})$, which represents the data generation rate in bps per unit area at location $\mathbf{z}$. The total generation rate of traffic of network is denoted by $w_{0}=\int_{A} r(\mathbf{z}) d x d y$, which is desired to be collected in $M$ information sinks. Assignment of network traffic to the sinks is such that $i^{t h}$ sink receives $w_{i}$, which we refer to as the weight of the sink, and accordingly we define a density function to model both sources and sinks:

$$
\rho(\mathbf{z})=r(\mathbf{z})-\sum_{i=1}^{M} w_{i} \delta\left(x-x_{i}\right) \delta\left(y-y_{i}\right)
$$

Note that $\rho(\mathbf{z})$ involves Dirac deltas for the spatial density of information rate at the locations of sinks. Obviously,
$\sum_{i=1}^{M} w_{i}=w_{0}$, which implies the total traffic collected by the sinks is equal to the total traffic generated in the network.

In the next step, we define a vector quantity that models flow of information. Let $\mathbf{D}(\mathbf{z})=\left(D_{x}, D_{y}\right)$ denote this vector field ${ }^{1}$ whose direction represents the direction of flow of information at point $\mathbf{z}=(x, y)$, and its magnitude represents the density of information rate passing through a line segment perpendicular to the direction of $\mathbf{D}(\mathbf{z})$. In other words, if we consider a line segment with a small length $\Delta l$ at $\mathbf{z}$ and perpendicular to the direction of $\mathbf{D}(\mathbf{z})$, the information crosses that line segment with rate $|\mathbf{D}(\mathbf{z})| \Delta l$. The above definition of $\mathbf{D}(\mathbf{z})$ implies that for a closed contour $C \in A$ we have:

$$
\oint_{C} \mathbf{D}(\mathbf{z}) \cdot \mathbf{d} \mathbf{n}=\int_{S(C)} \rho(\mathbf{z}) d x d y
$$

in which $\mathbf{d n}$ is a differential vector normal to the contour at each point of its boundary and pointing to the outside of the contour, the dot represents the inner product of vectors in two-dimensional space, and $S(C)$ is the area surrounded by the closed contour $C$. Equation (1) is analogous to Gauss' law in electrostatics theory, and it has a simple interpretation in our formulation: the rate at which information exits a contour is the net sum of the sources inside that contour. The following is known as Divergence Theorem in vector calculus:

$$
\oint_{C} \mathbf{D} \cdot \mathbf{d} \mathbf{n}=\int_{S(C)}\left(\frac{\partial D_{x}}{\partial x}+\frac{\partial D_{y}}{\partial y}\right) d x d y
$$

Equations (1) and (2) hold for an arbitrary contour $C$. This implies the following PDE form for information flow: ${ }^{2}$

$$
\nabla \cdot \mathbf{D}(\mathbf{z})=\frac{\partial D_{x}}{\partial x}+\frac{\partial D_{y}}{\partial y}=\rho(\mathbf{z})
$$

in which $x$ and $y$ are variables of a Cartesian coordinate frame in the network. In vector calculus, $\nabla \cdot$ is also known as divergence operator, and it gives the density of sources (sinks) of a vector field [7]. It is important to note that equation (3) is a representation of flow conservation law in continuous form.

The boundary condition of the above PDE is a result of the fact that information is not intended to exit the boundary of the network or enter it from the outside.

$$
D_{n}(\mathbf{z})=\mathbf{0} \forall \mathbf{z} \in \partial \mathbf{A}
$$

in which $D_{n}(\mathbf{z})$ is the normal component of $\mathbf{D}(\mathbf{z})$ along the boundary of $A$, and $\partial A$ represents the boundary of $A$. This condition is known as Neumann boundary condition.

An important note about the PDE of (3) and the boundary condition of (4) is that these conditions do not result in a unique value for $\mathbf{D}(\mathbf{z})$. The remaining issue is to decide what additional condition(s) to place on $\mathbf{D}(\mathbf{z})$ so that the resulting vector field generates a desirable set of routes. The intuition we follow is that by making $\mathbf{D}(\mathbf{z})$ as uniform as possible we obtain routes that cause the traffic to be highly dispersed throughout the network. In turn, this decreases both node congestion and collisions, and leads to high throughput.

\footnotetext{
${ }^{1}$ Throughout this paper, the boldface letters represent vector quantities.

${ }^{2}$ The operator $\nabla$ appears in different forms in many PDEs in the context of vector calculus. This operator is defined as $\nabla=\frac{\partial}{\partial x} \hat{i}+\frac{\partial}{\partial y} \hat{j}$, where $\hat{i}$ and $\hat{j}$ represent unit vectors along $x$ and $y$ axes respectively.
} 
Spreading the communication load can be accomplished by minimizing the following quadratic cost function:

$$
J(\mathbf{D})=\int_{A} K(\mathbf{z})|\mathbf{D}(\mathbf{z})|^{2} d x d y
$$

in which $K(\mathbf{z})$ takes into consideration the cost of routing through point $\mathbf{z}$ in the network. In [1], we have used $K(\mathbf{z})$ for energy efficient routing by assigning relatively high values to it in areas of the network where the sensors have low residual energies. Also, we showed that this form of cost function results in maximum spreading of the communication load among the network resources. It balances the communication load of the network in such a way that we avoid having a high load somewhere in the network while the resources are underutilized somewhere else. Moreover, it is shown in [3] that minimizing the above cost function minimizes the number of sensor nodes required to handle the total communication burden of the network. The summary of optimization problem and its constraints can be written as:

$$
\begin{array}{ll}
\text { Minimize } & J(\mathbf{D})=\int_{A} K(\mathbf{z})|\mathbf{D}(\mathbf{z})|^{2} d x d y \\
\text { s.t. } & \nabla \cdot \mathbf{D}(\mathbf{z})=\rho(\mathbf{z}) \quad \& \quad D_{n}(\mathbf{z})=0, \forall \mathbf{z} \in \partial A
\end{array}
$$

Our prior work includes two results regarding the optimization problem presented in (6). The first result gives the necessary and sufficient conditions for optimality of the cost function with a given assignment of communication load to the sinks, which implies fixed $w_{i}$ values. Our second result gives the necessary and sufficient conditions for optimality when the weights of the sinks are a part of the optimization problem, and we find optimal $w_{i}$ values for sinks.

The basic result: if $\mathbf{E}(\mathbf{z})=K(\mathbf{z}) \mathbf{D}(\mathbf{z})$, then the cost function in (6) is optimal if and only if $\operatorname{curl}^{3}$ of $\mathbf{E}(\mathbf{z})$ is zero:

$$
\nabla \times \mathbf{E}(\mathbf{z})=\left(-\frac{\partial E_{x}}{\partial y}+\frac{\partial E_{y}}{\partial x}\right) \hat{k}=0
$$

in which $\hat{k}$ is a unit vector normal to the network plane. Since $\nabla \times \mathbf{E}$ is zero, $\mathbf{E}$ is conservative, and it can be written as the gradient of a scalar potential function: $\mathbf{E}(\mathbf{z})=\nabla \mathbf{U}(\mathbf{z})$. This potential function satisfies:

$$
\nabla^{2} U(\mathbf{z})-\frac{\nabla \mathbf{K}(\mathbf{z}) \cdot \nabla \mathbf{U}(\mathbf{z})}{K(\mathbf{z})}=K(\mathbf{z}) \rho(\mathbf{z})
$$

When $K(\mathbf{z})$ is constant over the network, the potential function satisfies the Poisson's equation:

$$
\nabla^{2} U(\mathbf{z})=\frac{\partial^{2} U}{\partial x^{2}}+\frac{\partial^{2} U}{\partial y^{2}}=\rho(\mathbf{z})
$$

The combination of the divergence property, the zero-curl property, and the boundary conditions give $\mathbf{D}(\mathbf{z})$ uniquely. Note that boundary conditions of $\mathbf{D}$ imply that the gradient of $U$ is zero along the normal direction to the boundary.

\footnotetext{
${ }^{3}$ In the context of vector calculus, $\nabla \times$ is the two dimensional curl operator, whose value for a vector field $\mathbf{F}=\left[\begin{array}{ll}F_{x} & F_{y}\end{array}\right]$ is defined as: $\nabla \times \mathbf{F}(\mathbf{z})=$ $\left(-\frac{\partial F_{x}(\mathbf{z})}{\partial y}+\frac{\partial F_{y}(\mathbf{z})}{\partial x}\right) \hat{k}$, where $\hat{k}$ is a unit vector perpendicular to $\hat{i}$ and $\hat{j}$ (i.e., $\hat{k}=\hat{i} \times \hat{j}$ ). Curl operator gives the density of rotation of a vector field.
}

Optimizing weights of sinks: in this case the optimization is written as:

$$
\begin{array}{ll}
\text { Minimize } & J(\mathbf{D})=\int_{A} K(\mathbf{z})|\mathbf{D}(\mathbf{z})|^{2} d x d y \\
\text { s.t. } & \rho(\mathbf{z})=r(\mathbf{z})-\sum_{i=1}^{M} w_{i} \delta\left(x-x_{i}\right) \delta\left(y-y_{i}\right) \\
& \sum_{i=1}^{M} w_{i}=w_{0} \\
& \nabla \cdot \mathbf{D}(\mathbf{z})=\rho(\mathbf{z}) \quad \& \quad D_{n}(\mathbf{z})=0, \forall \mathbf{z} \in \partial A
\end{array}
$$

We solve this optimization by using the following result:

If the potential function at the locations of $M$ sinks is $U_{1}$, $U_{2}, \ldots, U_{M}$, then the necessary and sufficient conditions for optimality of the cost function in (8) are:

$$
\nabla \times \mathbf{E}(\mathbf{z})=0 \quad \& \quad U_{i}=U_{j}, \quad \forall 1 \leq i, j \leq M
$$

Additionally if we increase the weight of sink $i$ by a small amount $\epsilon$, and subtract that amount from the weight of sink $j$, then the increment in the cost function in (8) is:

$$
\Delta J=2 \epsilon\left(U_{i}-U_{j}\right)
$$

In section IV we use the above results to develop iterations that optimize the assignment of the network traffic to the sinks. Such iterations reduce the load assignment of the sinks that have a high potential value and assign more load to those with a lower potential value. In [10] we have shown that the iterations converge to optimal weights and hence optimal assignment of the network load to the sinks.

\section{Placement of The TRAFFiC SinKS}

So far we have assumed that the traffic sinks are fixed at their locations. In this section, we show how our vector field formulation gives an algorithm for relocating traffic sinks such that the steepest decrease in the cost function is achieved. Note that the steepest descent direction corresponds to the gradient of the cost function with respect to the location of each traffic sink. The following theorem gives the basis for a method to relocate the sinks for decreasing the cost function.

Theorem: Assume that the location of the traffic sink $i$ is $\mathbf{z}_{i}=$ $\left(x_{i}, y_{i}\right)$. If a small incremental change is made to the location of this traffic sink, and it is moved to $\left(x_{i}+\Delta x, y_{i}+\Delta y\right)$, the amount of change in the optimal value of the cost function given in (6) is:

$$
\Delta J=2 w_{i} \mathbf{E}\left(x_{i}, y_{i}\right) \cdot \mathbf{\Delta} \mathbf{z}
$$

where $\Delta \mathbf{z}=(\Delta x, \Delta y)$.

Proof: We use the following identities:

Identity 1: If $c$ is a scalar function and $\mathbf{F}$ is a vector field, then:

$$
\nabla \cdot(c \mathbf{F})=c \nabla \cdot \mathbf{F}+\mathbf{F} \cdot \nabla c
$$

Identity 2: If $A$ is a region in the plane with boundary $\partial A$, and $F$ is a vector field on $A$, then

$$
\int_{A} \nabla \cdot \mathbf{F} d x d y=\oint_{\partial A} \mathbf{F} \cdot \mathbf{d n}
$$

in which $\mathbf{d n}$ is the differential vector normal to the boundary of $A$ pointing outward. The second identity is the divergence theorem, which we also used it before in equation (2). 
Assume $\mathbf{D}(\mathbf{z}), \mathbf{E}(\mathbf{z}), U(\mathbf{z})$ and $\rho(\mathbf{z})$ represent the values of vector fields, the potential function and the density of sources for the case in which sink $i$ is at $\mathbf{z}_{i}$, and assume $\mathbf{D}^{\prime}(\mathbf{z}), \mathbf{E}^{\prime}(\mathbf{z})$, $U^{\prime}(\mathbf{z})$, and $\rho^{\prime}(\mathbf{z})$ represent the same quantities after we make a small change in the location of sink $i$ and move it to $\mathbf{z}_{i}+\Delta \mathbf{z}_{i}$. After this change, density of sources is:

$$
\begin{aligned}
& \rho^{\prime}(\mathbf{z})=\rho(\mathbf{z})+w_{i} \delta\left(x-x_{i}\right) \delta\left(y-y_{i}\right) \\
& -w_{i} \delta\left(x-x_{i}-\Delta x\right) \delta\left(y-y_{i}-\Delta y\right)
\end{aligned}
$$

Now we make the following definitions:

$$
\begin{aligned}
& \mathbf{D}^{\prime}(\mathbf{z})=\mathbf{D}(\mathbf{z})+\mathbf{d}(\mathbf{z}) \\
& \mathbf{E}^{\prime}(\mathbf{z})=\mathbf{E}(\mathbf{z})+\mathbf{e}(\mathbf{z})
\end{aligned}
$$

It is easy to verify that:

$$
\begin{aligned}
& \nabla \cdot \mathbf{d}(\mathbf{z})=\rho^{\prime}(\mathbf{z})-\rho(\mathbf{z})=w_{i} \delta\left(x-x_{i}\right) \delta\left(y-y_{i}\right)- \\
& w_{i} \delta\left(x-x_{i}-\Delta x\right) \delta\left(y-y_{i}-\Delta y\right)
\end{aligned}
$$

The change of the cost after moving the $i^{\text {th }}$ traffic sink is:

$$
\begin{aligned}
& \Delta J=J\left(\mathbf{D}^{\prime}\right)-J(\mathbf{D})= \\
& \int_{A} K(\mathbf{z})\left(\left|\mathbf{D}^{\prime}(\mathbf{z})\right|^{2}-|\mathbf{D}(\mathbf{z})|^{2}\right) d x d y= \\
& \int_{A} K(\mathbf{z})\left(|\mathbf{D}(\mathbf{z})+\mathbf{d}(\mathbf{z})|^{2}-|\mathbf{D}(\mathbf{z})|^{2}\right) d x d y= \\
& 2 \int_{A} K(\mathbf{z}) \mathbf{d}(\mathbf{z}) \cdot \mathbf{D}(\mathbf{z}) d x d y+\int_{A} K(\mathbf{z})|\mathbf{d}(\mathbf{z})|^{2} d x d y
\end{aligned}
$$

Since $(\Delta x, \Delta y)$ is very small in both components, we can ignore the second order term in the above equation. Hence:

$$
\begin{aligned}
& \Delta J=2 \int_{A} K(\mathbf{z}) \mathbf{d}(\mathbf{z}) \cdot \mathbf{D}(\mathbf{z}) d x d y= \\
& 2 \int_{A} \mathbf{d}(\mathbf{z}) \cdot \mathbf{E}(\mathbf{z}) d x d y
\end{aligned}
$$

Note that we have used $K(\mathbf{z}) \mathbf{D}(\mathbf{z})=\mathbf{E}(\mathbf{z})$. Now we use Identity 1 for $c=U(\mathbf{z})$ and $F=\mathbf{d}(\mathbf{z})$ :

$$
\nabla \cdot(U(\mathbf{z}) \mathbf{d}(\mathbf{z}))=U(\mathbf{z}) \nabla \cdot \mathbf{d}(\mathbf{z})+\mathbf{d}(\mathbf{z}) \cdot \nabla U(\mathbf{z})
$$

By using $\mathbf{E}(\mathbf{z})=\nabla U(\mathbf{z})$, the above equation is written as:

$$
\mathbf{d}(\mathbf{z}) \cdot \mathbf{E}(\mathbf{z})=\nabla \cdot(U(\mathbf{z}) \mathbf{d}(\mathbf{z}))-U(\mathbf{z}) \nabla \cdot \mathbf{d}(\mathbf{z})
$$

Substituting the above value in equation (13) leads us to:

$$
\begin{aligned}
& \Delta J=2 \int_{A} \nabla \cdot(U(\mathbf{z}) \mathbf{d}(\mathbf{z})) d x d y- \\
& 2 \int_{A} U(\mathbf{z}) \nabla \cdot \mathbf{d}(\mathbf{z}) d x d y
\end{aligned}
$$

Now we use the divergence theorem given in Identity 2 for $\mathbf{F}=U(\mathbf{z}) \mathbf{d}(\mathbf{z})$ We have:

$$
\int_{A} \nabla \cdot(U(\mathbf{z}) \mathbf{d}(\mathbf{z})) d x d y=\oint_{\partial A} U(\mathbf{z}) \mathbf{d}(\mathbf{z}) \cdot \mathbf{d} \mathbf{n}
$$

in which $\mathbf{d n}$ is a differential vector normal to $\partial A$ and pointing outward. Recall that the Neumann boundary condition states both $\mathbf{D}(\mathbf{z})$ and $\mathbf{D}^{\prime}(\mathbf{z})$ have zero components in the direction normal to the boundary of $A$. Hence, $\mathbf{d}(\mathbf{z})=\mathbf{D}^{\prime}(\mathbf{z})-\mathbf{D}(\mathbf{z})$ also has zero normal component at every point of the boundary of $A$. This causes the inner product in the integrand of equation (15) to be 0 . Therefore:

$$
\int_{A} \nabla \cdot(U(\mathbf{z}) \mathbf{d}(\mathbf{z})) d x d y=0
$$

On the other hand from equation (12) we have $\nabla \cdot \mathbf{d}(\mathbf{z})=$ $w_{i} \delta\left(x-x_{i}\right) \delta\left(y-y_{i}\right)-w_{i} \delta\left(x-x_{i}-\Delta x\right) \delta\left(y-y_{i}-\Delta y\right)$. Therefore:

$$
\begin{aligned}
& \int_{A} U(\mathbf{z}) \nabla \cdot \mathbf{d}(\mathbf{z}) d x d y= \\
& w_{i} \int_{A} U(\mathbf{z}) \delta\left(x-x_{i}\right) \delta\left(y-y_{i}\right) d x d y- \\
& w_{i} \int_{A} U(\mathbf{z}) \delta\left(x-x_{i}-\Delta x\right) \delta\left(y-y_{i}-\Delta y\right) d x d y \\
& =w_{i}\left(U\left(x_{i}, y_{i}\right)-U\left(x_{i}+\Delta x, y_{i}+\Delta y\right)\right)
\end{aligned}
$$

By substituting (17) and (16) in (14) we find:

$$
\Delta J=2 w_{i}\left(U\left(x_{i}+\Delta x, y_{i}+\Delta y\right)-U\left(x_{i}, y_{i}\right)\right)
$$

Now we use the fact that $\mathbf{E}(\mathbf{z})=\nabla U(\mathbf{z})$, hence if $\Delta x$ and $\Delta y$ are small values, then we can write:

$$
\begin{aligned}
& U\left(x_{i}+\Delta x, y_{i}+\Delta y\right)-U\left(x_{i}, y_{i}\right)= \\
& \mathbf{E}\left(x_{i}, y_{i}\right) \cdot(\Delta x \hat{i}+\Delta y \hat{j})=\mathbf{E}\left(x_{i}, y_{i}\right) \cdot \Delta \mathbf{z}
\end{aligned}
$$

By substituting (19) in (18):

$$
\Delta J=2 w_{i} \mathbf{E}\left(x_{i}, y_{i}\right) \cdot \Delta \mathbf{z}
$$

\section{QED.}

The above theorem yields the following results:

Corollary 1: The gradient of cost function $J$ with respect to the location of $i^{\text {th }}$ sink, $\mathbf{z}_{\mathbf{i}}$, can be written as:

$$
\frac{\partial J}{\partial \mathbf{z}_{\mathbf{i}}}=2 w_{i} \mathbf{E}\left(\mathbf{z}_{i}\right)
$$

Therefore, the direction of steepest descent of the cost function is the opposite of the gradient, i.e., $\mathbf{F}_{i}=-2 w_{i} \mathbf{E}\left(\mathbf{z}_{\mathbf{i}}\right)$.

We call $\mathbf{F}_{i}$ the force at the $i^{\text {th }}$ sink.

Corollary 2: The necessary condition for optimality of the cost function with respect to locations of sinks is that $\mathbf{E}\left(\mathbf{z}_{\mathbf{i}}\right)=0$ for all sinks.

Generally, because the density of the sources $\rho(\mathbf{z})$ has a Dirac delta form in the location of the traffic sinks, $\mathbf{E}(\mathbf{z})$ shows a high sensitivity to $\mathbf{z}$ when we are close to a sink. To avoid possible errors, we use an average of $\mathbf{E}(\mathbf{z})$ in the neighborhood of each traffic sink to find the steepest descent direction for relocation of that sink. This average is defined as:

$$
\mathbf{E}_{i}=\frac{1}{\left|S_{i}\right|} \int_{S_{i}} \mathbf{E}(\mathbf{z}) d x d y
$$

where $S_{i}$ is a small area containing the traffic sink $i$, and $\left|S_{i}\right|$ represents the area of $S_{i}$. Accordingly, we define force at the $i^{t h}$ sink as $\mathbf{F}\left(\mathbf{z}_{i}\right)=-2 w_{i} \mathbf{E}_{i}$. Relocation of sinks in the direction of steepest descent leads us to update the location of each sink in the following manner:

$$
\mathbf{z}_{i}^{j+1}=\mathbf{z}_{i}^{j}-\theta w_{i} \mathbf{F}_{i}\left(\mathbf{z}_{i}^{j}\right)
$$

where $\mathbf{z}_{i}^{j}$ is the location of the traffic sink $i$ at the $j^{\text {th }}$ iteration, and $\theta$ is a small step size. We stop the iterations when the maximum of the absolute values of $\mathbf{F}_{i}$ among all the traffic sinks is below a certain threshold.

An interesting note about the relocation algorithm is that the value of $\mathbf{F}_{i}$ is analogous to the force applied to a charged particle in electrostatics. Also, the cost function is analogous to the potential energy. Since force is always in the direction of reducing the potential energy, relocating the traffic sinks in its direction always reduces the cost function. In addition to force, the vector field model for the design of sensor networks has a one-to-one similarity to electrostatics quantities. Various aspects of this analogy are shown in Table I. The similarity with electrostatics gives many useful intuitions to solve sensor networking problems through analogies. However, despite the 214 
TABLE I

Sensor network analysis tool based on vector space vs. electrostatics

\begin{tabular}{c|c} 
Sensor Network & Electrostatics \\
\hline Load density $\mathbf{D}(\mathbf{z})$ & Electric displacement: $\overrightarrow{\mathbb{D}}$ \\
\hline $\mathbf{E}(\mathbf{z})$ & Electric field: $\overrightarrow{\mathbb{E}}=\frac{\mathbb{\mathbb { D }}}{\epsilon}$ \\
\hline$K(\mathbf{z})^{-1}$ & Dielectric Constant: $\epsilon$ \\
\hline$J(\mathbf{D})=$ & Potential Energy: \\
\hline$K(\mathbf{z})|\mathbf{D}(\mathbf{z})|^{2} d x d y$ & $e=\int \frac{1}{\epsilon} \overrightarrow{\mathbb{E}}^{2} d x d y$ \\
\hline$U(\mathbf{z})$ & Electric potential: $V$ \\
\hline$\rho(\mathbf{z})$ & Density of charges: $\rho$ \\
\hline $\int_{A}$ sink with weight $w_{i}$ & A Particle with Negative Charge $q$ \\
\hline Force: $\mathbf{F} i=-2 w_{i} \mathbf{E}\left(\mathbf{z}_{i}\right)$ & Force: $\overrightarrow{\mathbb{F}}=q \mathbb{\mathbb { E }}$ \\
\hline$\vec{\nabla} \cdot \mathbf{D}(\mathbf{z})=\rho(\mathbf{z})$ & Maxwell's equation: $\nabla \cdot \overrightarrow{\mathbb{D}}=\rho$ \\
\hline$\nabla \times \mathbf{E}(\mathbf{z})=0$ & Maxwell's equation: $\nabla \times \overrightarrow{\mathbb{E}}=0$
\end{tabular}

the Neumann boundary condition in our problem does not appear in electrostatics ${ }^{4}$ and therefore, most of the mathematical derivations in our problem need to be done independently and not through the analogy. For example, calculation of force applied to a particle with charge $q$ in electrostatics is done by removing that charge and calculating the electric field $\overrightarrow{\mathbb{E}}$ in absence of that charge, and finally the force is found through $\overrightarrow{\mathbb{F}}=q \overrightarrow{\mathbb{E}}$. Such a simple approach is not applicable in finding force in our problem since removing a sink with weight $w_{i}$ causes $\int_{A} \rho(\mathbf{z}) d x d y$ to become nonzero, which leads to a contradiction: using the Neumann boundary condition on the boundary of network implies that $\oint_{\partial A} \mathbf{D}(\mathbf{z}) \cdot \mathbf{d n}=0$ (because $\mathbf{D}(\mathbf{z})$ is tangent to the boundary of network at every point of it); on the other hand using Gauss' law on the boundary implies that $\oint_{A} \mathbf{D}(\mathbf{z}) \cdot \mathbf{d} \mathbf{n}=\int_{A} \rho(\mathbf{z}) d x d y \neq 0$. This contradiction shows that the problem after removing a sink does not have a solution. Fortunately, in most of practical cases, mature tools in vector calculus and PDEs help us perform analysis (similar to the one we did in this section to find force values) without a direct dependence on the analogy, while the analogy gives strong intuitions to use the right vector calculus tools and useful insights to the properties of interest.

\section{Summary of the Design Algorithm}

The results of the previous sections can be summarized as an algorithm that optimally partitions the network into territories of the traffic sinks and relocates the sinks in the direction of the steepest descent of the cost function. The design steps are:

1. Find the total amount of information of sources $w_{0}=$ $\int_{A} r(\mathbf{z}) d x d y$.

2. Assign the total load $w_{0}$ evenly among the traffic sinks: $w_{i}=w_{0} / M$ for $1 \leq i \leq M$.

3. Form $\rho(\mathbf{z})=r(\mathbf{z})-\sum_{i=1}^{M} w_{i} \delta\left(x-x_{i}\right) \delta\left(y-y_{i}\right)$

4. Numerically solve the PDE for potential function of equation (7).

5. If the values of the potential function at all the traffic sinks are the same (or all the pairwise differences are very small), the optimal weights for the given locations of the traffic sinks are found. Otherwise decrease the weights

\footnotetext{
${ }^{4}$ Boundary conditions in electrostatics are as follows: if the charge density along the boundary is zero, then the normal component of $\overrightarrow{\mathbb{D}}$ and the tangent component of $\overrightarrow{\mathbb{E}}$ are continuous on the two sides of each boundary point.
}

of the sinks that have a higher potential value than the average of $U\left(\mathbf{z}_{i}\right)$ among all sinks, and conversely, increase the weights of the sinks that have a smaller potential than the above average:

$$
\begin{aligned}
& \Delta w_{i}=-\gamma\left(U\left(\mathbf{z}_{i}\right)-\frac{1}{M} \sum_{i^{\prime}=1}^{M} U\left(\mathbf{z}_{i^{\prime}}\right)\right) \\
& w_{i}^{\prime}=w_{i}+\Delta w_{i}
\end{aligned}
$$

and go to step 3 .

The above algorithm gives the optimal values of the weights of the traffic sinks when their locations are fixed. Note that reassignment of weights in step 4 preserves the sum of weights of all sinks at constant $w_{0}$. If relocation of the traffic sinks for decreasing the cost function is a part of the optimization problem, the following step is added to the iterations:

6. Form force values, $\mathbf{F}_{i}\left(\mathbf{z}_{i}^{j}\right)=-2 w_{i} \mathbf{E}\left(\mathbf{z}_{i}^{j}\right)$, for all sinks. If the values of $\mathbf{F}_{i}\left(\mathbf{z}_{i}^{j}\right)$ are zero (or very small) for all sinks then stop; otherwise, relocate the sink by using,

$$
\mathbf{z}_{i}^{j+1}=\mathbf{z}_{i}^{j}+\theta \mathbf{F}\left(\mathbf{z}_{i}^{j}\right)
$$

and go to step 3 .

In the above algorithm, both $\gamma$ and $\theta$ are small nonnegative step sizes. The last step of the algorithm relocates sinks in the direction of the steepest descent of cost function. An issue of interest is to find the territories of the traffic sinks. At the end of the algorithm, the territories are found by following the flux lines of $\mathbf{E}(\mathbf{z})=\nabla U(\mathbf{z})$. A point $\mathbf{z}$ belongs to the territory of the traffic sink $i$ if the flux line starting at $\mathbf{z}$ terminates at the location of the traffic sink $i$. Note that the divergence property implies that only one flux line passes through every point that is not a traffic sink, and the flux lines can only terminate at the locations of a traffic sink. Therefore, each point resides in the territory of one of the traffic sinks.

\section{A Numerical EXAmple}

In this section we present a numerical example of a sensor network with multiple sinks. In this example we find the optimal weight and the territory for each traffic sink and use the direction of steepest descent of the cost function in order to relocate the sinks. The network is a $1 \times 1$ area, and we assume that the total load is 100 , and is uniformly distributed in the network, i.e., $r(\mathbf{z})=100$ for all $(\mathbf{z})$ in the network. Also we assume $K(\mathbf{z})=1$. We place 9 traffic sinks in the network at the following locations:

$$
\begin{array}{lll}
\mathbf{z}_{1}=(0.20,0.30) & \mathbf{z}_{2}=(0.40,0.35) & \mathbf{z}_{3}=(0.67,0.30) \\
\mathbf{z}_{4}=(0.25,0.60) & \mathbf{z}_{5}=(0.65,0.55) & \mathbf{z}_{6}=(0.85,0.55) \\
\mathbf{z}_{7}=(0.30,0.80) & \mathbf{z}_{8}=(0.60,0.78) & \mathbf{z}_{9}=(0.80,0.82)
\end{array}
$$

In the next step we divide the total load of 100 units evenly among the traffic sinks. This means that $w_{1}=w_{2}=$ $\ldots=w_{9}=w_{0} / 9=11.1$. Then, we solve the PDE for the potential function $U(\mathbf{z})$ numerically, and from it we find $\mathbf{D}(\mathbf{z})$. The resulting potential function is shown in Fig. 1-(a), the corresponding equipotential lines are shown in Fig. 1-(b). The values of the potential functions at the traffic sinks are: $U_{1}=0.0074, U_{2}=0.0201, U_{3}=0.0118, U_{4}=0.0233$, $U_{5}=0.0465, U_{6}=0.0430, U_{7}=0.0262, U_{8}=0.0485$, and $U_{9}=0.0543$. The total value of the cost function in this 


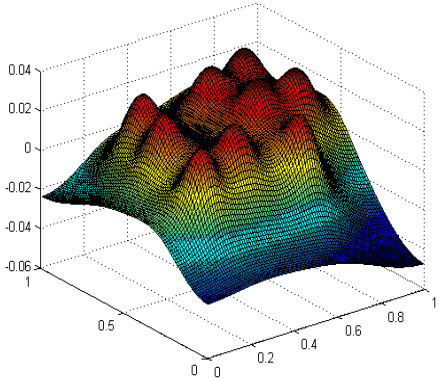

(a)

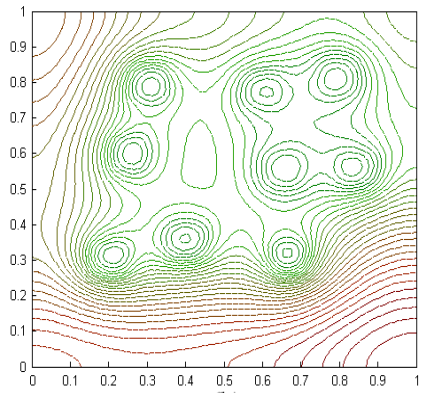

(b)
Fig. 1. (a) The value of the potential function $U(\mathbf{z})$ for the case with 9 traffic sinks. (b) The equipotential lines of $U(\mathbf{z})$

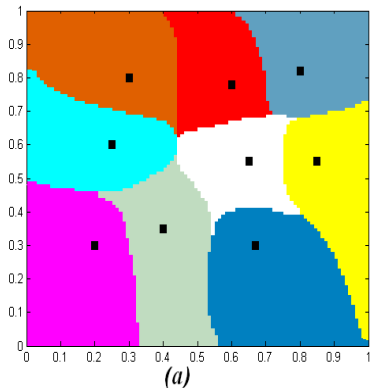

(a)

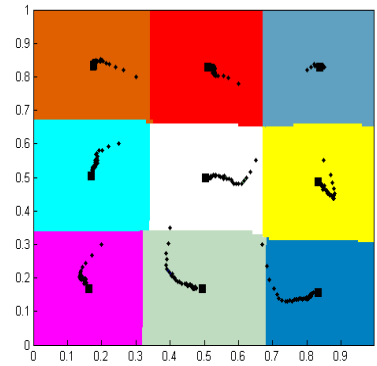

(b)
Fig. 2. (a) The territories for different traffic sinks before applying steepest relocation iterations based on force. (b) Placement of sinks and their corresponding territories after applying steepest relocation iterations based on force. The dark dots show the location of the traffic sinks during the iterations that use the, and the small dark squares show the final placement of the sinks.

case is 1.81. Since the values of the potential function are not the same at all the locations of the sinks, we know that we can update the weights of the sinks to further reduce the cost function. In the next step, we use the iterations in Section IV to update the weights in order to optimize the cost function with respect to the weights of the sinks. The calculations show that the algorithm converges to the weight values within 1 percent of the optimal weights in 3 iterations:

$$
\begin{array}{lllll}
w_{1}=14.1 & w_{2}=11.4 & w_{3}=15.5 & w_{4}=11.5 & w_{5}=7.8 \\
w_{6}=9.9 & w_{7}=12.2 & w_{8}=8.8 & w_{9}=8.7
\end{array}
$$

With the above values of weights, the cost function reduces to 1.09 , which shows about $66 \%$ decrease. We have used the gradient step size $\gamma=200$. Also, the values of the potential function at the traffic sinks were calculated to be $U_{1}=\ldots=$ $U_{9}=0.0281$. The territories for the 9 traffic sinks at the end of these iterations are shown in Fig. 2-(a).

In the last step we use force values to iteratively relocate each sink in the direction of steepest descent of the cost function. At each iteration of relocating sinks, we use the iterations that balance the load among sinks and make the potential value at locations of all of them equal. We continue iterations until all sinks have zero value for force and all of them have equal potential. The results show that the final placement of the sinks are centers of nine even squares partitioning the network into a chessboard-like pattern. With this placement, the cost function reduces to 0.73 , which shows a $33 \%$ improvement in the cost function compared to the previous case. The territory of each sink is one of the squares. The placement of the sinks, the resulting territories after relocating iterations, and the traces of sinks during steepest descent relocations are shown in Fig. 2- (b). Although the resulting territories and optimal placement of the traffic sinks were predictable from the symmetry of this problem, the result shows that relocating along force values can successfully find the optimal placement of the sinks.

\section{CONCLUSION}

In this paper, we presented a mathematical formulation based on vector space analysis in order to study several design aspects of wireless sensor networks. We defined a conservative vector field related to the communication load in the network and expressed it as the gradient of a scalar function. In an optimal assignment of the network load to the sinks, the value of potential function at the locations of all sinks should be equal. Based on this fact, we gave iterations that lead us to the optimal partitioning of network into territories of sinks. Additionally, we showed that the steepest descent in the cost function is achieved by relocating each sink in the direction of a force value, which is proportional to the value of the vector field at the location of that sink. We used this result to introduce iterations that relocate sinks until it finds a placement in which the vector field is zero at location of all sinks. The cost function keeps decreasing during such iterations. It should be remarked that iterations for relocating of sinks continue until force is zero at location of all sinks. Although in all of the numerical examples that the authors have studied the iterations lead to a globally optimal placement of sinks, this fact demand future research. It needs to be investigated under what conditions the placement after relocation iterations is a global optimum, and if the algorithm may be trapped in local optima, under what conditions a trapping is likely to happen. As a final remark, in this paper we assumed that the traffic received by the sinks is equal to the total traffic generated in the network and date is not aggregated along the paths. Studying the flow models under data aggregation needs to be considered in future extensions of this works.

\section{REFERENCES}

[1] M. Kalantari and M.A. Shayman. Energy efficient routing in sensor networks. In Conference on Information Sciences and Systems, 2004.

[2] M. Kalantari and M.A. Shayman. Routing in wireless ad hoc networks by analogy to electrostatic theory. In IEEE International Communications Conference, 2004.

[3] S. Toumpis and L. Tassiulas. Efficient node deployment in massively dense sensor networks as an electrostatics problem. In IEEE Infocom, 2005.

[4] S. Toumpis and L. Tassiulas. Optimal deployment of large wireless sensor networks. IEEE Transactions on Information Theory, 52(7):29352953, 2006.

[5] Stig Larsson and Vidar Thome. Partial Differential Equations with Numerical Methods. Springer, 2003.

[6] Jrgen Jost. Partial differential equations. Springer, 2002.

[7] B. R. McQuistan. Scalar and Vector Fields: A Physical Interpretation. John Wiley \& Sons, 1965.

[8] J.D. Jackson. Classical Electrodynamics. John Wiley \& Sons, 1999.

[9] D.K. Cheng. Field and Wave Electromagnetics. Addison-Wesley, 1989.

[10] M. Kalantari and M.A. Shayman. Design optimization of multisink sensor networks by analogy to electrostatic theory. In Wireless Communication and Networking Conference, 2006. 